\title{
On Solution of Min-Max Composition Fuzzy Relational Equation
}

\author{
N.M. Niama*
}

Date of acceptance 20/5/2007

\begin{abstract}
In this paper, Min-Max composition fuzzy relation equation are studied. This study is a generalization of the works of Ohsato and Sekigushi. The conditions for the existence of solutions are studied, then the resolution of equations is discussed.
\end{abstract}

\section{Introduction}

The concept of fuzzy relational equations introduced by Sanchez [5], is a generalization of well known Boolean equations.

Let $A$ and $B$ be two fuzzy sets of two finite spaces $X, Y$ respectively and $R$ a fuzzy relation of the set $\mathrm{X} \times \mathrm{Y}$. Consider the following fuzzy relation equation $R \circ \mathrm{A}=B \ldots$ (1)

Where "o" is the Min-Max composition.

Speaking with terminology of systems theory, $A$ and $B$ represent a class of fuzzy inputs and a class of fuzzy equation (1).

In this paper, we illustrate other algorithms, to solve equation (1).

\section{Preliminaries}

Let $I=[0,1]$ be the real unite interval and we set for every real numbers $a, b \in I$,

$\bar{a}=1-a, a \wedge b=\min \{a, b\}, a \vee b=\max \{a, b\}$

, [1] of course, we have

$1-\overline{a \vee b}=\bar{a} \wedge \bar{b}, \quad \overline{a \wedge b}=\bar{a} \vee \bar{b}$

(De Morgan's laws) $2-(a \vee b) \wedge c=(a \wedge c) \vee(b \wedge c)$,

$(a \wedge b) \vee c=(a \vee c) \wedge(b \vee c)$

[distributivity laws]

Let $\mathrm{X}=\left\{x_{1}, x_{2}, \ldots, x_{n}\right\}, \mathrm{Y}=\left\{y_{1}, y_{2}, \ldots, y_{m}\right\}$ be finite sets, $F(\mathrm{X})=\left\{\mathrm{A}: \mu_{\mathrm{A}}: \mathrm{X} \rightarrow I\right\}$ the set of all fuzzy sets of $X$.

$I_{r}=\{1,2,3, \ldots, r\}$ the set of first $r$ natural numbers.

Following Zedeh's [1,2], we remember that $F(\mathrm{X})$ is a complete distributive lattice with the pointwise operations defined for every $x_{i} \in X, \quad i \in I_{n} \quad$ as

1- $\mathrm{A}\left(x_{i}\right)=1-\mathrm{A}\left(x_{i}\right)$

$2-(\mathrm{A} \wedge B)\left(x_{i}\right)=\mathrm{A}\left(x_{i}\right) \wedge B\left(x_{i}\right), \quad(\mathrm{A} \vee B)\left(x_{i}\right)=\mathrm{A}\left(x_{i}\right) \vee B\left(x_{i}\right)$

3 - $(\mathrm{A} \circ B)\left(x_{i}\right)=\mathrm{A}\left(x_{i}\right) \circ B\left(x_{i}\right)$

4- $(\mathrm{A} \circ B)^{-1}\left(x_{i}\right)=B^{-1}\left(x_{i}\right) \circ \mathrm{A}^{-1}\left(x_{i}\right)$

and a natural ordering as

$\mathrm{A} \leq B \quad$ iff $\quad \mathrm{A}\left(x_{i}\right) \leq B\left(x_{i}\right) \quad$ where

$\mathrm{A}, B \in F(X)$

Let $x_{i} \in X, y_{j} \in Y, i \in I_{n}$ and $j \in I_{m}$, we recall the following definitions:

*College of Science for Women, Baghdad University 


\section{Definition 1.[1]}

A fuzzy relation $R$ between two finite sets $X$ and $Y$ is a mapping from the Cartesian product of crisp set $X, Y$ to the unite interval [0,1] (an element of $F(\mathrm{X} \times \mathrm{Y}))$

\section{Definition 2.[6]}

\section{Fuzzy}

relation

equation is given in the form of which the composite of fuzzy input $\mathrm{A}$ and fuzzy relation $R$ equals fuzzy output $B$. The input $A$ and the output $B$ are fuzzy sets represented by $\mathrm{A}=\left\{a_{i}\right\}, \quad B=\left\{b_{j}\right\}$ respectively. The fuzzy relation $R$ represents the causality of input and output.

\section{Definition 3.[1]}

Let $A$ be the set of all possible vectors $\mathrm{A}=\left[a_{i} \mid i \in I_{n}\right]$ such that $a_{i} \in[0,1]$ for all $i \in I_{n}$ and let a partial ordering on $A$ be defin as follows:

For any pair ${ }^{1} \mathrm{~A},{ }^{2} \mathrm{~A} \in \mathrm{A}$, ${ }^{1} \mathrm{~A} \leq{ }^{2} \mathrm{~A}$ if and only if ${ }^{1} a_{i} \leq^{2} a_{i}$ for all $i \in I_{n}$

\section{Definition 4.[3,4]}

An element $\hat{A}$ of $S(R, B)$ is called a maximal solution of Eq.(1), if for all $\mathrm{A} \in S(R, B), \quad \mathrm{A} \geq \hat{\mathrm{A}}$ implies $\mathrm{A}=\hat{\mathrm{A}}$

It is well established that whenever the solution set $S(R, B) \neq \varphi, \quad$ it is always contains a unique maximal solution , $\hat{A}$.

\section{Definition 5.[4,5]}

An element $\stackrel{\vee}{ }$ of $S(R, B)$ is called minimal solution of Eq.(1) if for all $\mathrm{A} \in S(R, B), \quad \mathrm{A} \leq \stackrel{\mathrm{A}}{ }$ implies $\mathrm{A}=\stackrel{\mathrm{A}}{ }$ and when $S(R, B) \neq \varphi$ it may contain several minimal solutions.

\section{Existence of solutions}

We now establish some theorems concerning the existence of solutions of the equation $\overline{R_{\circ} \mathrm{A}}=B$ where " $\bar{\circ}$ " denotes a min-max composition of two binary operators $G$ and $\Gamma$, maps from $L^{2}$ in $L$ where $L^{2}=[0,1] \times[0,1], L$ the interval $[0,1]$, the $\mathrm{O}$ or $\overline{\mathrm{O}}$ compositions will be particular cases of the $G-\Gamma$ composition introduced here.

More precisely, if the operator $G$ is associative and with the notation $G\left(a_{1}\right)=a_{1}$ and, for $m \geq 2, \underset{j \in[1, m]}{G}\left(a_{j}\right)=G\left(\underset{j \in[1, m-1]}{G}\left(a_{j}\right), a_{m}\right)$ , Eq.(1) can be written: $\forall i \in\{1,2, \ldots, m\}, \underset{j \in J}{G}\left(\Gamma\left(r_{i j}, a_{j}\right)\right)=b_{i} \quad J=\{1,2,3, \ldots, n\}$ ...(4)

Furthermore, if we suppose that $(x, y) \mapsto G(x, y)$ is a monotone non decreasing mapping on $L^{2}$ and $x \mapsto \Gamma(x, y)$ is a monotone non decreasing mapping on $L$ for every $y \in L$ then $\forall r_{i j} \in L, \quad \underset{j \in J}{G}\left(\Gamma\left(0, a_{j}\right)\right) \leq \underset{j \in J}{G}\left(\Gamma\left(r_{i j}, a_{j}\right)\right) \leq \underset{j \in J}{G}\left(\Gamma\left(1, a_{j}\right)\right)$

This leads us to state the necessary condition of the following theorem, in which $E(G, \Gamma, R)$ denotes the set of all solutions of Eq.(1) when the relation $R$ is supposed unknown. This set is simply designated by $E(G, \Gamma)$. 


\section{Theorem 1}

Let $G$ and $\Gamma$ be two functions from $L^{2}$ into $L$ such that, for every $x, y, z$ and $t$ belongs to $L$,

(i) $G(G(x, y), z)=G(x, G(y, z))$

(ii) $(x, y)<(z, t) \Rightarrow G(x, y) \leq G(z, t)$

(iii) $x<z \Rightarrow \Gamma(x, y) \leq \Gamma(z, y)$

if the set $E(G, \Gamma)$ is non-empty,

then $\left.\forall i \in\{1,2, \ldots, m\}, b_{i} \in \underset{j \in \in J}{G}\left(\mathrm{r}\left(0, a_{j}\right)\right){ }_{j \in J}^{G}\left(r\left(1, a_{j}\right)\right)\right]$

...(5)

conversely if condition (5) is fulfilled and if moreover:

(iv) the function $G:(x, y) \mapsto G(x, y)$ is continuous on $L^{2} \ldots(6)$

(v) the function $x \mapsto \Gamma(x, y)$ is continuous on $L$ for each $y \in L \ldots$ (7) then $E(G, \Gamma)$ is nonempty.

Indeed, if conditions (5)-(7) are fulfilled, then $t_{i j} \in\left[\Gamma\left(0, a_{j}\right), \Gamma\left(1, a_{j}\right)\right]$ exists such that $\underset{j \in J}{G}\left(t_{i j}\right)=b_{i}$, and $r_{i j} \in[0,1]$ exists such that $t_{i j}=\Gamma\left(r_{i j}, a_{j}\right)$, hence $E(G, \Gamma)=\varphi$

\section{Corollary 1}

If the set $E(0, \overline{0})$ is nonempty then $\max b_{i} \leq \underset{j \in J}{0}\left(a_{j}\right)$, where $\quad I=\{1,2, \ldots, m\} \ldots$ (8). Conversely if (8) holds and if (i) $(x, y) \mapsto 0(x, y) \quad$ is continuous on $L^{2}$, (ii) $x \mapsto \overline{0}(x, y)$ is continuous on $L$ for each $y \in L$, then $E(0, \overline{0}) \neq \varphi$ In the same way, for $G=\overline{0}$ and $\Gamma=0$, we obtain:

\section{Corollary 2}

If the set $E(0, \overline{0})$ is nonempty then $\min _{i \in I} b_{i} \geq \overline{0}\left(a_{j \in J}\right) \ldots$ (9)

Conversely if (9) holds and if:

(i) $(x, y) \mapsto \overline{0}(x, y)$ is continuous on $L^{2}$,

(ii) $x \mapsto 0(x, y)$ is continuous on $L$ for each $y \in L$, then $E(0, \overline{0}) \neq \Phi$.

That is: $R \circ \mathrm{A}=B \quad$ iff $0\left(\overline{0}\left(r_{i j}, a_{j}\right)\right)=b_{i}$ for all $i$ in $I$ and $\quad \stackrel{-}{\circ \circ} \mathrm{A}=B$ iff $\underset{j \in J}{0}\left(0\left(r_{i j}, a_{j}\right)\right)=b_{i}$ for all $i$ in $I$.

\section{Theorem 2}

The equation $\Lambda .\left(R^{-} \mathrm{A}\right)=B$ ...(10) has solutions if and only if $\forall i \in I$. $\lambda_{i} \underset{j \in J}{\overline{0}}\left(a_{j}\right) \leq b_{i} \leq \lambda_{i}$

From now $R_{i}, \lambda_{i}$ and $b_{i}$ will indicate the $i$ th row of $R, \Lambda$ and $B$. Where $\Lambda$ is the column matrix with coefficients $\Lambda=\left(\lambda_{i}\right) \in \mu$ (is the fuzzy matrix).

\section{Proof of theorem 2}

If $\lambda_{i} \neq 0, \quad$ Eq.(10) is equivalent to $\forall i \in I, R_{i}^{\circ}{ }^{-} \mathrm{A}=\frac{b_{i}}{\lambda_{i}}$. This equation has solutions if and only if (corollary 2) $\underset{j \in J}{-}\left(a_{j}\right) \leq \frac{b_{i}}{\lambda_{i}} \leq 1$, i.e if and only if $b_{i} \in\left[\lambda_{i} \underset{j \in J}{\bar{O}}\left(a_{j}\right), \lambda_{i}\right]$. This condition is also true for $\lambda_{i}=0$. 


\section{Theorem 3}

Let $\mathfrak{R}$ be the set of solutions of fuzzy relation equation $\quad \overline{R \circ A}=B, \quad$ then $\mathfrak{R}=\left\{R:\right.$ fuzzy $\quad$ relation $\left.\mid R_{\circ}^{-} \mathrm{A}=B\right\} \neq \varphi$ iff $A^{-1} \psi B \in \mathfrak{R}$.

$$
\text { If } \mathfrak{R} \neq \phi \text {, then } \bar{R}=\mathrm{A}^{-1} \psi B
$$

is greatest element in $\mathfrak{R}$.

Existence condition for theorem 3: The necessary and sufficient condition for the set $\mathfrak{R} \neq \varphi$ is:

There exists $a, j \in J_{n}$ such that $a_{j} \geq b_{k}$ for all $\kappa \in \mathrm{K}_{p}$.

This existence condition has been documented by pedrycz[7] for the Max-Min composition.

\section{Resolution to fuzzy relational equation}

$$
\text { Let } \quad R \in F(\mathrm{X} \times \mathrm{Y}) \quad \text { and }
$$
$\mathrm{A} \in F(\mathrm{X}) \quad$ we define $R \circ \mathrm{A}=B \ldots(1), \quad B \in F(\mathrm{Y}), \quad$ the min-max composition of $R$ and $\mathrm{A}$ as

$$
B\left(y_{i}\right)=\overbrace{j=1}^{m} \underset{i=1}{\wedge}\left[R\left(x_{i}, y_{i}\right), \mathrm{A}\left(x_{i}\right)\right] \ldots(
$$

Let the membership matrices of $\mathrm{A}, R$ and $B$ denoted by $\mathrm{A}=\left[a_{i}\right], R=\left\lfloor r_{i j} \mid, B=\left\lfloor b_{j}\right]\right.$, respectively, where $a_{i}=\mu_{\mathrm{A}}\left(x_{i}\right), r_{i j}=\mu_{R}\left(x_{i}, y_{j}\right), b_{j}=\mu_{B}\left(y_{j}\right)$ for all $i \in I_{n}$ and $j \in J_{m}$.

This mean that all the entries in the matrices $A$.

$R$ and $B$ are real numbers in unite interval $I=[0,1]$, when matrices $\mathrm{A}$ and $R$ are given and matrix $B$ is to be determined from Eq.(1) the problem is trivial. It is solved simply by performing the Min-Max composition-like operation on A and $R$ as defined by Eq.(2). Clearly the solution in this case exists and is unique.

\section{Example 1}

\section{Given}

$$
R=\left[\begin{array}{ccc}
.5 & .8 & .3 \\
.9 & .2 & .4 \\
.7 & .6 & .9 \\
0 & .9 & 1
\end{array}\right]_{4 \times 3} \text { and } \mathrm{A}=\left[\begin{array}{c}
.7 \\
.5 \\
1
\end{array}\right]_{3 \times 1}
$$

Determine the solution of $\overline{R \circ} A=B$ from Eq.(2)

$$
\begin{aligned}
& B\left(y_{j}\right)=\underset{j=1}{\stackrel{m}{\wedge}}\left\{\underset{i=1}{\stackrel{n}{\vee}}\left(\mu_{R}\left(x_{i}, y_{i}\right), \mu_{\mathrm{A}}\left(x_{i}\right)\right)\right\} \\
& B\left(y_{1}\right)=\underset{j=1}{m}\{\underbrace{n}_{i=1}\left(\mu_{R}\left(x_{i}, y_{1}\right), \mu_{\mathrm{A}}\left(x_{i}\right)\right)\}=\wedge(.7,8,1)=.7 \\
& B\left(y_{2}\right)=\wedge(.9, .5,1)=.5 \\
& B\left(y_{3}\right)=\wedge(.7, .6,1)=.6 \\
& B\left(y_{4}\right)=\wedge(.7, .9,1)=.7 \\
& \text { Then } B=\left[\begin{array}{l}
.7 \\
.5 \\
.6 \\
.7
\end{array}\right]
\end{aligned}
$$

The problem becomes far from trivial when one of two matrices on the left-hand side of Eq.(1) is unknown. In this case, the solution is neither guaranteed to exist nor to be unique. Since $B$ in Eq.(1) is obtained by composing $\mathrm{A}$ and $R$, it is suggestive to view the problem of determining A from $B$ and $R$ as a decomposition of $B$ with respect to $R$. Let us assume that a pair of specific matrices $B$ and $R$ from Eq.(1) is given and that we wish to determine the set of 
all particular matrices of the form

A that satisfy Eq.(1).

Let each particular matrix A that satisfies Eq.(1) be called its solution and let $S(R, B)=\left\{A: \overline{R_{\circ} \mathrm{A}}=B\right\}$ denote the set of all solutions, (the solution set).

It follows immediately that when we take the inverse of both sides of Eq.(1) we will get:

$(R \circ \overline{\mathrm{A}})^{-1}=B^{-1}$

$\mathrm{A}^{T} \circ R^{T}=B^{T}$.

note that:

$\mathrm{A}^{-1}=\mathrm{A}^{T}, B^{-1}=B^{T} \quad$ and $\quad R^{-1}=R^{T}$ that is $R^{-1}(x, y)=R(y, x)=R^{T}$ thus $\mathrm{A}^{T}=\left[a_{i}: i \in I_{n}\right], \quad B^{T}\left[b_{j}: j \in J_{m}\right]$ and $R^{T}\left[r_{j, i}: i \in I_{n}, j \in J_{m}\right]$

Now we can solve Eq.(3) more simply than Eq.(1). Then if $\min _{i \in I_{n}} r_{j i}<b_{j}$ then values $a_{i} \in[0,1]$ exist that satisfy Eq.(3) and, matrix $\mathrm{A}^{T}$ exists that satisfies the matrix equation thus $S\left(R^{T}, B^{T}\right) \neq \phi$. When $S\left(R^{T}, B^{T}\right) \neq \varphi$, the maximum solution $(\hat{\mathrm{A}})^{T}=\left(a_{i}, i \in I_{n}\right)$ of Eq.(3) is determined by:

$\hat{a_{i}}=\max \left(\sigma\left(r_{j, i}, b_{j}\right)\right)$

where $\sigma\left(r_{j, i}, b_{j}\right)=\left[\begin{array}{ccc}b_{j} & \text { if } & r_{j, i} \leq b_{j} \\ 1 & \text { if } & r_{j, i}>b_{j}\end{array}\right.$

We next determine the set $\stackrel{\vee}{S}\left(R^{T}, B^{T}\right)$ of its minimal solutions of Eq.(3) can be determined by the following procedure:

1- Determine the sets

$J_{j}\left((\hat{\mathrm{A}})^{T}\right)=\left\{i \in I_{n}: \max \left(r_{j, i}, a_{i}\right)=b_{j}\right\}$

for

all $j \in J_{m}$ and then construct

their product denote elements of

$J\left((\hat{\mathrm{A}})^{T}\right)=\prod_{j \in J_{m}} J_{j}\left((\hat{\mathrm{A}})^{T}\right) J\left((\hat{\mathrm{A}})^{T}\right)$ by

$\beta=\left(\beta_{j}: j \in J_{m}\right)$

2- For each $\beta \in J\left((\hat{\mathrm{A}})^{T}\right)$ and each $i \in I_{n}$ determine the set $\mathrm{K}(\beta, i)=\left\{j \in J_{m}: \beta_{j}=i\right\}$

3- For each $\beta \in J\left((\hat{\mathrm{A}})^{T}\right)$ generate the n-tuple $g(\beta)=\left(g_{i}(\beta): i \in I_{n}\right)$ by taking $g_{i}(\beta)=\left[\begin{array}{lll}\max _{j \in \kappa(\beta, i)} b_{j} & \text { if } & \mathrm{K}(\beta, i) \neq \varphi \\ 0 & \text { if } & \mathrm{K}(\beta, i)=\varphi\end{array}\right.$ 4- From all the n-tuples $g(\beta)$ generated in step (3) select all the maximum ones and $(\widehat{\mathrm{A}})^{T} \neq(\hat{\mathrm{A}})^{T}$ by pairwise composition.

The resulting set of ntuples is the set $\stackrel{\vee}{S}\left(R^{T}, B^{T}\right)$ of the minimal solution of Eq.(3). Finally the solution set $S\left(R^{T}, B^{T}\right)$ is fully characterized by the maximum and minimal solutions in the following sense:

It consists exactly of the maximum solution $(\hat{A})^{T}$, all the minimal solutions and all elements of $A$ that are between $(\hat{\mathrm{A}})^{T}$ and each of the minimal solution.

Formally

$$
\left.S\left(R^{T}, B^{T}\right)=\underset{(\vee \mathrm{A}}{\mathrm{A}}\right)^{T}<(\hat{\mathrm{A}})^{T},(\hat{\mathrm{A}})^{T}>
$$


Where the union is taken for all $(\mathrm{\vee}){ }^{T} \in \stackrel{\vee}{S}\left(R^{T}, B^{T}\right)$. We got the set of solutions of Eq.(3). We must now take the transpose of $(\hat{\mathrm{A}})^{T}$ and each of the minimal solutions $(\hat{\mathrm{A}})^{T}$ that is $\left((\hat{\mathrm{A}})^{T}\right)^{T}=\hat{\mathrm{A}}$ the maximal solutions of Eq.(1). And $\left((\check{\mathrm{A}})^{T}\right)^{T}=\check{\mathrm{A}}$ the minimal solution of Eq.(1).

$$
\text { So } s(R, B)=\underset{A}{\underset{V}{V}}<\underset{\mathrm{A}, \hat{\mathrm{A}}>}{\vee}
$$

Example 2; Given

$$
R=\left[\begin{array}{ccc}
.2 & 1 & .4 \\
0 & .6 & .3 \\
0 & 1 & .3
\end{array}\right] \quad B=\left[\begin{array}{c}
.5 \\
.5 \\
.5
\end{array}\right]
$$

Determine all solutions of

$$
R_{\circ}^{-} \mathrm{A}=B
$$

$\underline{\text { Sol }}$

Take the inverse of the equation above $\left(\stackrel{-}{R^{-} \mathrm{A}}\right)^{-1}=B^{-1} \Rightarrow \mathrm{A}^{T} \circ R^{T}=B^{T} \ldots$

Where

$$
\begin{gathered}
R^{T}=\left[\begin{array}{ccc}
.2 & 0 & 0 \\
1 & .6 & 1 \\
.4 & 1 & .3
\end{array}\right] \quad B^{T}=\left[\begin{array}{lll}
.5 & .5 & .5
\end{array}\right]=\left[\begin{array}{lll}
b_{1} & b_{2} & b_{3}
\end{array}\right] \\
\mathrm{A}^{T}=\left[a_{1}, a_{2}, a_{3}\right]
\end{gathered}
$$

Thus, we first must find the solutions of Eq.(1). So we must determine whether $S\left(R^{T}, B^{T}\right)=\phi$, or not by:

First we determine whether $S(R, B)=\phi$ or not, by:

$$
\begin{aligned}
& \operatorname{Min}(.2,1, .4)=.2<.5=b_{1} \\
& \operatorname{Min}(0, .6, .3)=0<.5=b_{2} \\
& \operatorname{Min}(0,1, .3)=0<.5=b_{3}
\end{aligned}
$$

Thus $\quad S\left(R^{T}, B^{T}\right) \neq \phi \quad$ [now since $\left.S\left(R^{T}, B^{T}\right) \neq \phi\right]$. We determine the maximum solution $(\hat{\mathrm{A}})^{T}$ of Eq.(1) by:

$\hat{a_{1}}=\operatorname{Max} \sigma\left(r_{j, 1}, b_{j}\right)=\operatorname{Max}(.5, .5, .5)=.5$

$\hat{a_{2}}=\operatorname{Max} \sigma\left(r_{j, 2}, b_{j}\right)=\operatorname{Max}(1,1,1)=1$

$\hat{a_{3}}=\operatorname{Max} \sigma\left(r_{j, 3}, b_{j}\right)=\operatorname{Max}(.5, .5, .5)=.5$

$(\hat{\mathrm{A}})^{T}=(.5,1, .5)$. we can easily satisfy $(\hat{\mathrm{A}})^{T} \in S\left(R^{T}, B^{T}\right)$

$(\hat{\mathrm{A}})^{T}{ }^{ }{ }^{-} R^{T}=B^{T}$

$\left[\begin{array}{lll}.5 & 1 & .5\end{array}\right] \circ\left[\begin{array}{ccc}.2 & 0 & 0 \\ 1 & .6 & 1 \\ .4 & 1 & .3\end{array}\right]=\left[\begin{array}{lll}.5 & .5 & .5\end{array}\right]$

$\left[\begin{array}{lll}.5 & .5 & .5\end{array}\right]=\left[\begin{array}{lll}.5 & .5 & .5\end{array}\right], \quad$ hence $S\left(R^{T}, B^{T}\right) \neq \phi$

Next we apply the four steps of the procedure for determining the set $\stackrel{\vee}{S}\left(R^{T}, B^{T}\right)$ of all minimal solution of this reduced matrix equattion:

1- Employing the maximum solution $(\hat{\mathrm{A}})^{T}=\left(\begin{array}{lll}.5 & 1 & .5\end{array}\right)$ of the reduced equation, we obtain $J_{1}\left((\hat{\mathrm{A}})^{T}\right)=\left\{i \in I_{n}: \operatorname{Max}\left(r_{1, i}, \hat{a}_{i}\right)=b_{1}\right\}=\{1,3\}$
$J_{2}\left((\hat{\mathrm{A}})^{T}\right)=\left\{i \in I_{n}: \operatorname{Max}\left(r_{2, i}, \hat{a}_{i}\right)=b_{2}\right\}=\{1,3\}$
$J_{3}\left((\hat{\mathrm{A}})^{T}\right)=\left\{i \in I_{n}: \operatorname{Max}\left(r_{3, i}, \hat{a}_{i}\right)=b_{3}\right\}=\{1,3\}$ hence $J\left((\hat{\mathrm{A}})^{T}\right)=\prod J_{j}\left((\hat{\mathrm{A}})^{T}\right)=\{1,3\} \times\{1,3\} \times\{1,3\}$

$=\left\{\left(\begin{array}{lll}1 & 1 & 1\end{array}\right),\left(\begin{array}{llll}1 & 1 & 3\end{array}\right),\left(\begin{array}{llll}1 & 3 & 1\end{array}\right),\left(\begin{array}{llll}1 & 3 & 3\end{array}\right),\left(\begin{array}{llll}3 & 1 & 1\end{array}\right),\left(\begin{array}{llll}3 & 1 & 3\end{array}\right),\left(\begin{array}{llll}3 & 3\end{array}\right),\left(\begin{array}{lll}3 & 3\end{array}\right)\right\}$ 
2- The sets $\mathrm{K}(\beta, i)$ that we must determine for all $\beta \in J\left((\hat{\mathrm{A}})^{T}\right)$ and all $i \in I_{n}$ are

listed in the following table:

\begin{tabular}{|c|c|c|c|}
\hline \multicolumn{4}{|c|}{$\mathrm{K}(\beta, i)$} \\
\hline$\beta$ & $i=1$ & 2 & $g(\beta)$ \\
\hline$\left(\begin{array}{lll}1 & 1 & 1\end{array}\right)$ & $\{1,2,3\}$ & $\phi \quad \phi$ & $\left(\begin{array}{lll}.5 & 0 & 0\end{array}\right)$ \\
\hline$\left(\begin{array}{lll}1 & 1 & 3\end{array}\right)$ & $\{1,2\}$ & $\phi \quad\{3\}$ & $\left(\begin{array}{lll}.5 & 0 & .5\end{array}\right)$ \\
\hline$\left(\begin{array}{lll}1 & 3 & 1\end{array}\right)$ & $\{1,3\}$ & $\phi \quad\{2\}$ & $\left(\begin{array}{lll}.5 & 0 & .5\end{array}\right)$ \\
\hline$\left(\begin{array}{lll}1 & 3 & 3\end{array}\right)$ & $\{1\}$ & $\phi \quad\{2,3\}$ & $\left(\begin{array}{lll}.5 & 0 & .5\end{array}\right)$ \\
\hline$\left(\begin{array}{lll}3 & 1 & 1\end{array}\right)$ & $\{2,3\}$ & $\phi \quad\{1\}$ & $\left(\begin{array}{lll}.5 & 0 & .5\end{array}\right)$ \\
\hline$\left(\begin{array}{lll}3 & 1 & 3\end{array}\right)$ & $\{2\}$ & $\phi \quad\{1,3\}$ & $\left(\begin{array}{lll}.5 & 0 & .5\end{array}\right)$ \\
\hline$\left(\begin{array}{lll}3 & 3 & 1\end{array}\right)$ & $\{3\}$ & $\phi \quad\{1,2\}$ & $\left(\begin{array}{lll}.5 & 0 & .5\end{array}\right)$ \\
\hline$\left(\begin{array}{lll}3 & 3 & 3\end{array}\right)$ & $\phi$ & $\phi \quad\{1,2,3\}$ & $\left(\begin{array}{lll}0 & 0 & .5\end{array}\right)$ \\
\hline
\end{tabular}

3- For each $\beta \in J\left((\hat{\mathrm{A}})^{T}\right)$, we generate the triples $g(\beta)$ which are also listed in table above.

4- One of the triples $g(\beta)$ in table above is minimal solution $\left(\begin{array}{lll}.5 & 0 & .5\end{array}\right)$. Hence $\stackrel{\vee}{S}\left(R^{T}, B^{T}\right)=\left\{(\vee \mathrm{A})^{T}=\left(\begin{array}{lll}.5 & 0 & .5\end{array}\right)\right\}$ so we have the set of solution of equation (1) as

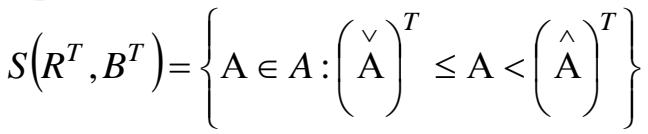

So to determine the solutions of equation $A \circ R=B$, we must take the transpose of $(\hat{\mathrm{A}})^{T},(\mathrm{\textrm {A }})^{T}$, that is $\left((\hat{\mathrm{A}})^{T}\right)^{T}=\left[\begin{array}{l}.5 \\ 1 \\ .5\end{array}\right]$

and

$$
\left((\mathrm{A})^{T}\right)^{T}=\left[\begin{array}{l}
.5 \\
0 \\
.5
\end{array}\right]
$$

Now the set $S(R, B)$ of all solution of the given matrix equation is now fully captured by the maximum solution $\hat{\mathrm{A}}=\left[\begin{array}{c}.5 \\ 1 \\ .5\end{array}\right]$ and the minimal solution

$$
\mathrm{A}=\left[\begin{array}{l}
5 \\
0 \\
.5
\end{array}\right]
$$

So we have:

$$
S(R, B)=\{\mathrm{A} \in A: \stackrel{\vee}{\mathrm{A}} \leq \mathrm{A} \leq \hat{\mathrm{A}}\}
$$

\section{Example 3}

$$
\text { Given } R=\left[\begin{array}{ll}
.2 & .3 \\
.4 & .5
\end{array}\right], \quad B=\left[\begin{array}{l}
.9 \\
.9
\end{array}\right]
$$

Determine all solutions of $R \circ \mathrm{A}=B$

\section{Sol}

Take the inverse of the equation above

so $((R \circ \mathrm{A})=B)^{-1}$

$(R \circ \mathrm{A})^{-1}=B^{-1}$

$\rightarrow \mathrm{A}^{T} \circ R^{T}=B^{T}$

where $R^{T}=\left[\begin{array}{ll}.2 & .4 \\ .3 & .5\end{array}\right], \quad B^{T}=\left[\begin{array}{ll}.9 & .9\end{array}\right]$, $\mathrm{A}^{T}=\left[\begin{array}{ll}a_{1} & a_{2}\end{array}\right]$

We first must find the solutions of Eq.(1). So we must determine whether $S\left(R^{T}, B^{T}\right)=\phi$, or not by:

First we determine whether $S(R, B)=\phi$ or not by: 
$\min (.2, .3)=.2<.9$

$\min (.4, .5)=.4<.9$

Thus $S\left(R^{T}, B^{T}\right) \neq \phi$

We determiner the maximum solution $(\hat{\mathrm{A}})^{T}$ of Eq.(1) by:

$\hat{a_{1}}=\max \sigma\left(r_{j, 1}, b_{j}\right)=\max (.9,9)=.9$

$\hat{a_{2}}=\max \sigma\left(r_{j, 2}, b_{j}\right)=\max (.9,9)=.9$

$(\hat{\mathrm{A}})^{T}=[.9,9] \in S\left(R^{T}, B^{T}\right), \quad$ hence $S\left(R^{T}, B^{T}\right) \neq \varphi$

Next we apply the four steps of the procedure for determining the set $\stackrel{\vee}{S}\left(R^{T}, B^{T}\right)$ for all minimal solution of this reduced matrix equation:

1- We obtain

$J_{1}\left((\hat{\mathrm{A}})^{T}\right)=\left\{i \in I_{n}: \max \left(r_{j, 1}, \hat{a}_{1}\right)=b_{j}\right\}=\{1,2\}$

$J_{2}\left((\hat{\mathrm{A}})^{T}\right)=\left\{i \in I_{n}: \max \left(r_{j, 2}, \hat{a_{2}}\right)=b_{j}\right\}=\{1,2\}$

hence $J\left((\hat{\mathrm{A}})^{T}\right)=\prod^{J}\left((\hat{\mathrm{A}})^{T}\right)=\{1,2\} \times\{1,2\}$

$=\{(1,1) \quad(1,2) \quad(2,1) \quad(2,2)\}$

2- The sets $\mathrm{K}(\beta, i)$ that we must determine for all $\beta \in J\left((\hat{\mathrm{A}})^{T}\right)$ and all $i \in I_{n}$ are

listed in the following table:

\begin{tabular}{|c|c|c|c|}
\hline \multicolumn{4}{|c|}{$\mathrm{K}(\beta, i)$} \\
\hline$\beta$ & $i=1$ & 2 & $g(\beta)$ \\
\hline$\left(\begin{array}{ll}1 & 1\end{array}\right)$ & $\{1,2\}$ & $\phi$ & $\left.\begin{array}{ll}(.9 & 0\end{array}\right)$ \\
\hline$\left(\begin{array}{ll}1 & 2\end{array}\right)$ & $\{1\}$ & $\{2\}$ & $\left(\begin{array}{ll}.9 & .9\end{array}\right)$ \\
\hline$\left(\begin{array}{ll}2 & 1\end{array}\right)$ & $\{2\}$ & $\{1\}$ & $\left(\begin{array}{ll}.9 & .9\end{array}\right)$ \\
\hline$\left(\begin{array}{ll}2 & 2\end{array}\right)$ & $\phi$ & $\{1,2\}$ & $\left.\begin{array}{ll}(0 & .9\end{array}\right)$ \\
\hline
\end{tabular}

3- For each $\beta \in J\left((\hat{\mathrm{A}})^{T}\right)$, we generate the triples $g(\beta)$ which are also listed in table above.

4- Two of the triples $g(\beta)$ in table above are minimal solutions $\quad\left(\begin{array}{ll}.9 & 0\end{array}\right),\left(\begin{array}{ll}0 & .9\end{array}\right)$

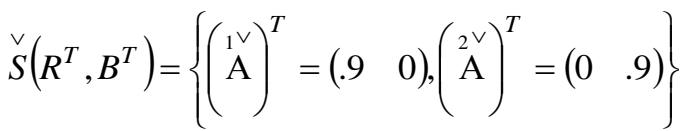

So we have the set of solution of equation

$$
\begin{gathered}
S\left(R^{T}, B^{T}\right)=\left\{\mathrm{A} \in A:(\stackrel{\mathrm{A}}{\mathrm{A}})^{T} \leq \mathrm{A} \leq(\hat{\mathrm{A}})^{T}\right\} \\
\cup\left\{\mathrm{A} \in A:\left(\begin{array}{c}
2 \vee \\
\mathrm{A}
\end{array}\right)^{T} \leq \mathrm{A} \leq(\hat{\mathrm{A}})^{T}\right\}
\end{gathered}
$$

So $S(R, B)=\bigcup_{\mathrm{A}}(\stackrel{\mathrm{A}}{\mathrm{A}}, \hat{\mathrm{A}})$.

Basic procedure to determine all solutions of the equation $R \circ \bar{\circ}=B$...(1)

1- Take the inverse (transpose) of both sides of Eq.(1) this results in the new equation $\mathrm{A}^{T} \circ R^{T}=B^{T} \ldots(2)$

2- If $\min _{i \in I_{n}} r_{j, i}<b_{j}$ then the equation has solution $S\left(R^{T}, B^{T}\right) \neq \varphi$ and the procedure terminates, otherwise proceed to step 3.

3- Determine $(\hat{A})^{T}$ by procedure 1 .

4- If $(\hat{\mathrm{A}})^{T}$ is not a solution of Eq.(2), then the equation has no solution, $S\left(R^{T}, B^{T}\right)=\varphi$.

5- Determine all minimal solutions of the reduced equation 
(3) by procedure 2: this results in

$$
\stackrel{\vee}{S}\left(R^{T}, B^{T}\right) \text {. }
$$

6- Determine the solution set of the reduced equation (3):

$$
S\left(R^{T}, B^{T}\right)=\bigcup_{\left(\begin{array}{c}
v \\
\mathrm{~A}
\end{array}\right)^{T}}\left((\hat{\mathrm{A}})^{T},(\hat{\mathrm{A}})^{T}\right) \text { where the }
$$

union is taken over all $(\stackrel{\vee}{\mathrm{A}})^{T} \in \stackrel{\vee}{S}\left(R^{T}, B^{T}\right)$.

7- Take the transpose of $(\mathrm{A})^{T}$ and each of the minimal

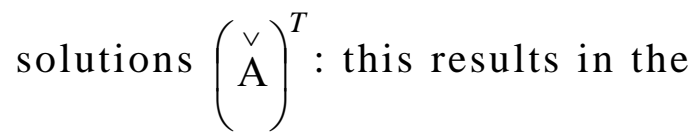
solution set $S(R, B)$ which is $S(R, B)=\bigcup_{\mathrm{A}}(\mathrm{A}, \hat{\mathrm{A}})$

\section{Procedure (1)}

From

the vector

$(\hat{\mathrm{A}})^{T}=\left(\hat{a_{i}}, i \in I_{n}\right)$ in which $\hat{a_{i}}=\max \sigma\left(r_{j, i}, b_{j}\right)$ where

$$
\sigma\left(r_{j, i}, b_{j}\right)=\left[\begin{array}{ccc}
b_{j} & \text { if } & r_{j, i} \leq b_{j} \\
1 & \text { if } & r_{j, i}>b_{j}
\end{array}\right.
$$

\section{Procedure (2)}

1 - Permute elements of $B^{T}$ and the corresponding columns of $R^{T}$ appropriately to arrange them in decreasing order.

2- Determine the set

$$
J_{j}\left((\hat{\mathrm{A}})^{T}\right)=\left\{i \in I_{n}: \max \left(\hat{a}_{i}, r_{j, i}\right)=b_{j}\right\}
$$

for all $j \in J_{m}$ and then construct their cartesian product $J\left((\hat{\mathrm{A}})^{T}\right)=\prod_{j \in J_{m}} J_{j}\left((\hat{\mathrm{A}})^{T}\right)$.
3- For each $\beta \in J\left((\hat{\mathrm{A}})^{T}\right)$ and each $i \in I_{n}$ determine the set $\mathrm{K}(\beta, i)=\left\{j \in J_{m}: \beta=i\right\}$.

4- For each $\beta \in J\left((\hat{\mathrm{A}})^{T}\right)$ generate the n-tuple $g(\beta)=\left(g_{i}(\beta): i \in I_{n}\right) \quad$ by taking

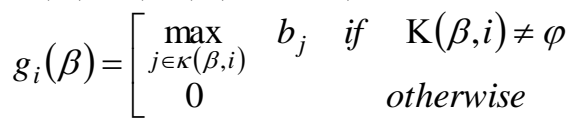

5 - From all the n-tuples $g(\beta)$ generated in step 4 select only the minimal ones, this results in $\stackrel{\vee}{S}\left(R^{T}, B^{T}\right)$

Note 1: if $R_{1}, R_{2}$ and $R_{3}$ are fuzzy relation on $\mathrm{X} \times \mathrm{Y}, \mathrm{Y} \times \mathrm{Z}$ and $\mathrm{X} \times \mathrm{Z}$, respectively. Where $R_{1} \circ R_{2}=R_{3} \quad$ is a fuzzy relational equation where " $\mathrm{O}$ " is the Max-Min product composition. If $R_{2}$ is unknown in Eq. $R_{1} \circ R_{2}=R_{3}$ we can find the maximal solution by the Eq. $\hat{R}_{2}=R_{1}^{T} \circ R_{3}$ where $R_{1}^{T}(x, y)=R_{1}(y, x)=R_{1}(y, x)$

Example: given $R_{1}=\left[\begin{array}{lll}.7 & .5 & 1 \\ .4 & 0 & .9\end{array}\right], R_{3}=\left[\begin{array}{lll}1 & .6 & .5 \\ .9 & .6 & .5\end{array}\right]$ Determine maximal solution of $R_{1} \circ R_{2}=R_{3}$

\section{Sol}

$$
\begin{aligned}
& \hat{R_{2}}=R_{1}^{T} \circ R_{3}=\left[\begin{array}{cc}
.7 & .4 \\
.5 & 0 \\
1 & .9
\end{array}\right] \circ\left[\begin{array}{lll}
1 & .6 & .5 \\
.9 & .6 & .5
\end{array}\right]=\left[\begin{array}{ccc}
.7 & .6 & .5 \\
.5 & .5 & .5 \\
1 & .6 & .5
\end{array}\right] \\
& \text { We can easily } \text { prove } \\
& \hat{R_{2}} \in S\left(R_{1}, R_{3}\right) \\
& R_{1} \circ \hat{R}_{2}=R^{3}
\end{aligned}
$$




$$
\begin{gathered}
R_{1} \circ \hat{R_{2}}=\left[\begin{array}{lll}
.7 & .5 & 1 \\
.4 & 0 & .9
\end{array}\right] \circ\left[\begin{array}{lll}
.7 & .6 & .5 \\
.5 & .5 & .5 \\
1 & .6 & .5
\end{array}\right] \\
=\left[\begin{array}{lll}
1 & .6 & .5 \\
.9 & .6 & .5
\end{array}\right]=R^{3}
\end{gathered}
$$

Note 2: If $A$ and $B$ are two fuzzy sets, respectively and $R$ a fuzzy relation of the set $\mathrm{X} \times \mathrm{Y}$. $R \bullet \mathrm{A}=B \quad$ where "• $\quad \operatorname{Max}$ product composition

$$
B\left(g_{i}\right)=(R \bullet \mathrm{A})\left(y_{i}\right)={\underset{j}{j=1}}_{\mathrm{m}}^{m}\left[R\left(x_{i}, y_{j}\right) \bullet \mathrm{A}\left(x_{i}\right)\right]_{i=1}^{n}
$$

if $\mathrm{A}$ is unknown we can

find the solution by equation

$$
\mathrm{A}=R^{T} \bullet B
$$

\section{Example}

$$
\text { Given } R=\left[\begin{array}{ccc}
3 & 5 & .8 \\
0 & .7 & 1 \\
.4 & .6 & .5
\end{array}\right], B=\left[\begin{array}{c}
.8 \\
1 \\
.5
\end{array}\right]
$$

\section{Sol}

We must find the solution:

$$
\begin{aligned}
& \mathrm{A}=R^{T} \bullet B=\left[\begin{array}{lll}
.3 & 0 & .4 \\
.5 & .7 & .6 \\
.8 & 1 & .5
\end{array}\right] \cdot\left[\begin{array}{l}
.8 \\
1 \\
.5
\end{array}\right] \\
& a_{1}=\max (.24 \quad 0 \quad .20)=.24 \\
& a_{2}=\max \left(\begin{array}{lll}
.4 & .7 & .3
\end{array}\right)=.7 \\
& a_{3}=\max \left(\begin{array}{lll}
.64 & 1 & .25
\end{array}\right)=1
\end{aligned}
$$$$
\therefore \mathrm{A}=\left[\begin{array}{c}
24 \\
.7 \\
1
\end{array}\right]
$$

to prove $\mathrm{A} \in S(R, B)$ we must prove $R \bullet \mathrm{A}=B$

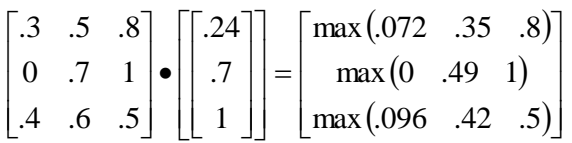

$$
\begin{aligned}
& =\left[\begin{array}{c}
.8 \\
1 \\
.5
\end{array}\right]=B
\end{aligned}
$$

\section{References}

1. Daruan, , 2000. Intelligent Hybrid Systems: Fuzzy Logic, Neural Network and Genetic Algorithms Belgian Nuclear Research Center (SCK. CEN) Mol, Belgium.

2. George J.K, , 1997.Fuzzy set foundation and applications Utest, Clair, Boyuou.

3. Klir, Folger A., 1994. "Fuzzy set uncertainty and information", Univ of New York.

4. Malik D.S. and John N., 1991 . Fuzzy relations on groups, fuzzy sets and systems, North Holland 43:117-123.

5. Sankar K Majumder D.K., 2001 "Fuzzy mathematical approach to pattern recognition" Calcutta, India.

6. Ohsato A. and Sekiguchi T., 1994.Combined form of fuzzy relational equations and its application, Proc. Int. Systems Man Cybornet., Bombay : 294299.

7. Pedrycz W., 1996 Processing in relational structures: Fuzzy relational equations, Fuzzy sets and systems 40 .

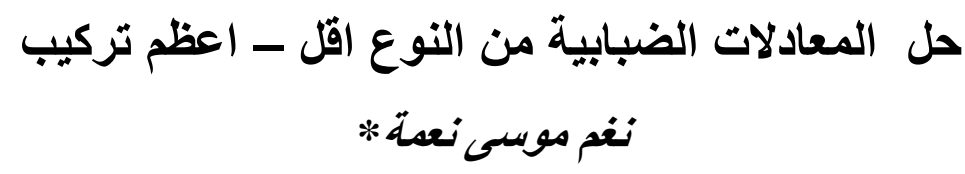

الم.م. جامعة بغداد- كلية العلوم للبنات

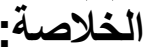

يتضمن البحث دراسة نوع من المعادلات الضبابية، هذه الدراسة مكملة ومتممة لدراسة كم من Ohsato . and Sekiguchi

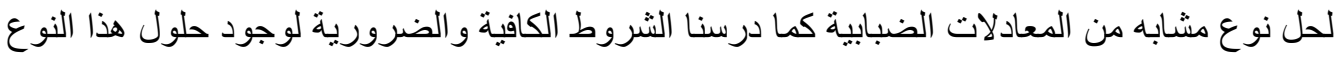
من المعادلات الضبابية ثم قدمنا خوارزمية جديدة لحل هذا النوع من المعادلات الضباتية الضبابة. 